\section{Easier immunoperoxidase staining with labour saving incubator box}

IM MUIR, AI ALEXANDER Department of Surgery, University of Melbourne, St Vincent's Hospital, Victoria, Australia

Immunoperoxidase staining methods are widely used in research laboratories, ${ }^{1}$ and more recently they have become established as a valuable tool in clinical pathology. ${ }^{23}$ Multiple incubation with immunoglobulins confers sensitivity, but also makes the tests labour intensive, particularly when large numbers of specimens are to be processed. The most time consuming and frustrating part of processing a large number of sections is the change from one antibody to the next. Our present use of immunoperoxidase is in staining sections of several hundred breast carcinomas using monoclonal antibodies. Five incubations are needed and total change over time for 33 sections amounts to one hour. We have developed an incubator box that greatly reduces the time and tedium of this procedure by eliminating handling slides between incubations. We have not found reference to similar apparatus in published reports.

\section{Description and use of the box}

The equipment consists of an acrylic box $(590 \mathrm{~mm} \times$ $330 \mathrm{~mm} \times 110 \mathrm{~mm}$ ) with lid and three slide irrigating bars (figs 1 and 2). The irrigating bars are of square section with a central hollow (fig lc) and are $575 \mathrm{~mm}$ long. A length of acrylic dowel cemented into the ends of the bars allows them to be suspended horizontally in slots cut in the sides of the box. The dowel at one end of the bar is drilled throughout its length (fig 1a) to permit entry of irrigating fluid from a 10 litre container. Clips on the underside of the irrigating bars (fig 1c) permit attachment of slides that can be flooded with irrigating fluid via a small channel, which communicates with the central hollow. Each bar holds 16 slides with the clips spaced at $36 \mathrm{~mm}$ centres. The clips are chromium plated, size 2 , spring clips with one limb removed and are attached by plated self tapping screws. Flow to the irrigating bar is controlled by the roller tap from an intravenous giving set. The irrigating bar can be set in three positions by a simple latch mechanism at the opposite end to the fluid inlet (fig le). An upright position allows slides to be mounted and air to be displaced from the system. The hor-

Accepted for publication 21 May 1986 izontal position permits irrigation of the sections and incubation with reagents. The dependent position permits irrigation fluid to be drained from the slide. A $4 \mathrm{~mm}$ hole in one corner of the box permits drainage of spent irrigation fluid.

The slides are set in the bars after blocking endogenous peroxidase. To rinse the slides they must be flooded with irrigation fluid, drained, flooded again and left in the horizontal position for five minutes and the process repeated. The slides are then drained and while in the dependent position the lower end of the slide is touched with a rolled up tissue to absorb most of the remaining fluid. The slides are then placed in the horizontal position. If the whole slide is still wetted in continuity with the moisture on the section the section can be isolated using a small camel hair brush soaked in acetone, thus permitting reagents to be localised on the section rather than spreading over the slide. Mounting sections towards one end of the slides rather than in the centre facilitates this. After the chromogen stage the slides are replaced in the carrier for counterstaining, dehydration, and clearing. Initially phosphate buffered saline (PBS) was used as the irrigating fluid, but we found that $0.9 \%$ sodium chloride in tap water gives indistinguishable results. Bovine serum albumin in phosphate buffered saline remains the best diluent for the immunoglobulins.

\section{Assessment of the box}

Many hundreds of sections have been stained with the new equipment, and overall, results have been excellent. We can process 48 sections at a time and still save 40 minutes on the time previously taken for $33 \mathrm{sec}$ tions. To assess formally the new equipment 40 pairs of slides were simultaneously processed, one from each pair in the traditional box and the other in the new staining box. The same batches of reagents were used for both boxes. After processing each slide of the pair was given a randomisation code before scoring by three independent observers. The first slide of each pair was then assessed for intensity of staining and rated as staining better, the same, or worse than its counterpart. The randomisation code was then broken and the non-parametric sign test applied to the data.

Of the 40 pairs of slides, 39 pairs were available for comparison, one section having been lost in the traditional box. Of the 117 pair assessments, 60 pairs were scored the same, in 35 the traditional box was preferred, and in 22 the new box was preferred. The sign test applied to this data gave a two tailed $p$ value of 0.25 , indicating no significant difference in the quality of staining from the boxes.

Finally, a small experiment was set up to test the effect of acetone on the staining protocol. Five pairs 


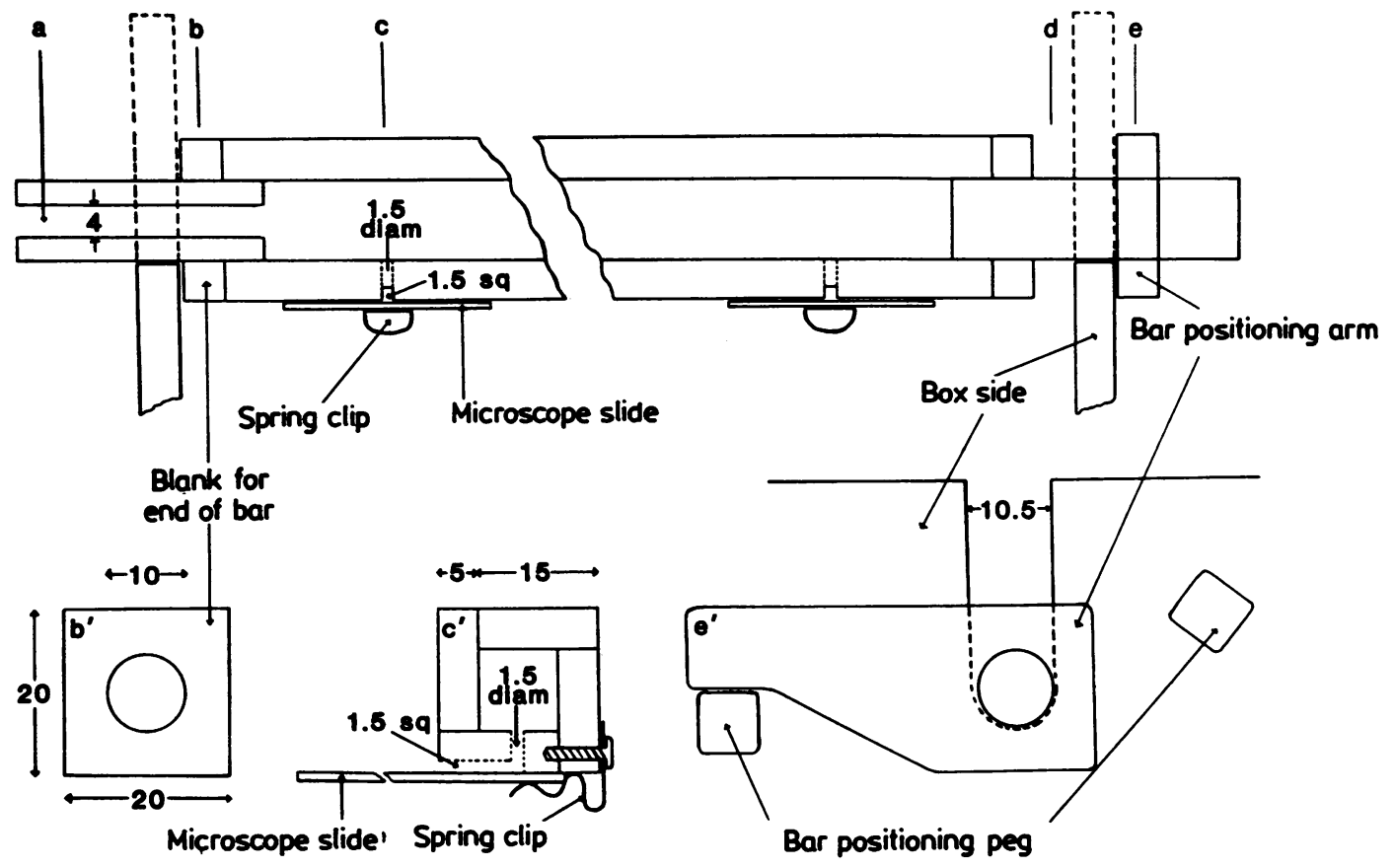

Fig 1 Slide irrigating bar: $b^{\prime}$ and $c^{\prime}$ are cross sections of the bar at $b$ and $c$, respectively; $e^{\prime}$ is side elevation of the box at $e$. Bar positioning arm in $e^{\prime}$ is shown resting on positioning peg which maintains slides in horizontal position; other peg maintains upright position, no peg is necessary to hold dependent position. Space at d permits horizontal movement of bar to right and disengages bar positioning arm from bar positioning pegs. Fluid enters bars at a and flows onto slide through channel at $c$. (All dimensions in $\mathrm{mm}$ ).

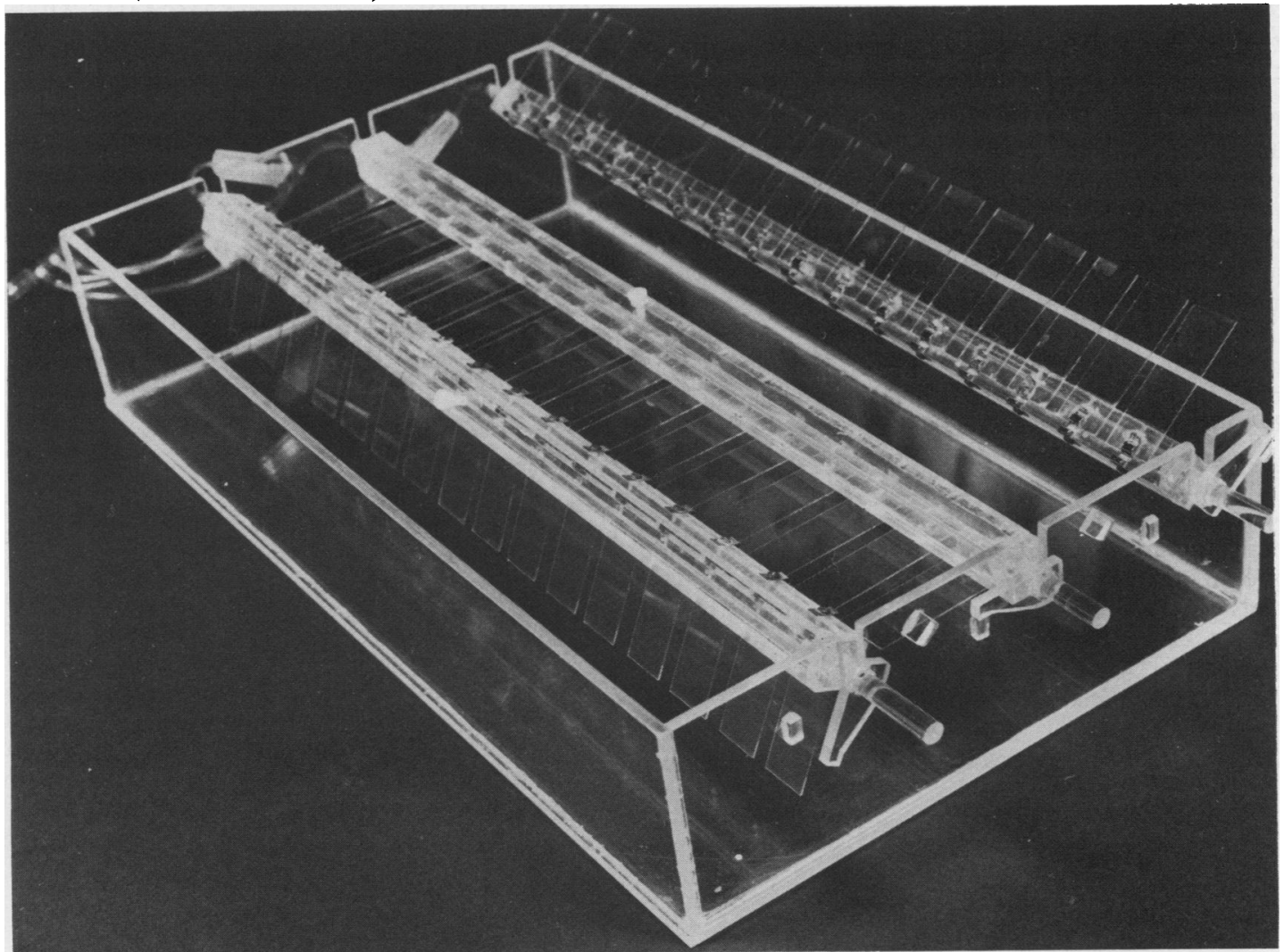

Fig 2 New incubator box (lid not shown) with front row of slides dependent, middle row horizontal, and back row upright. Positioning latch mechanism is shown in foreground; irrigating fluid enters bars at far end of box. 
of slides were used, one of each pair being processed with the usual diluent and the other in diluent containing $10 \%$ acetone. Acetone had no effect on the quality of staining.

\section{Discussion}

The results we obtained with the new equipment indicate that it can greatly reduce the time taken to process large numbers of slides, while retaining a high degree of quality. The equipment could be constructed for any number of slides, but is increasingly worthwhile when a dozen or more slides are processed. Readily available materials have been used, and the design has been aimed at simplicity of construction that is well within the capabilities of an average hospital carpentry department.

Other advantages are the rinsing of chromogens of dubious safety before touching the slides and the loss of fewer sections from the slides. We believe that the

\section{Microwave polymerisation of epoxy resin: rapid processing technique in ultrastructural pathology}

\section{ALC MCLAY, JDANDERSON, W MCMEEKIN From the University Department of Pathology, Glasgow Royal Infirmary, Glasgow, Scotland}

The problem of rapid processing in biological electron microscopy has often been addressed, particularly in relation to diagnostic pathology. Overnight processing schedules may now be regarded as normal, but although considerably shortened schedules are possible, our experience suggests that some loss of quality is evident when total processing times with standard epoxy resins are less than about three hours. Using conventional oven techniques, the polymerisation step, traditionally one of the longer elements in the process, needs to be at least half an hour. Recently there has been increasing interest in the use of microwave irradiation for tissue fixation, ${ }^{12}$ but although its use in dental practice ${ }^{3}$ and industry ${ }^{4}$ has been addressed, its possible application to polymerisation in electron microscopy seems to have been ignored. This paper shows that microwave polymerisation is readily practicable and that it may have distinct advantages over conventional procedures.

\section{Material and methods}

All tissue in this study was fixed in $4 \%$ phosphate usefulness of this equipment will find wide appeal among clinical pathologists and research workers.

We are grateful to Julio Micalessi and Rudi Rob of the works department, St Vincent's Hospital, for their expert help in accurately cutting the acrylic and to $\mathrm{Mr}$ Dominic Persano of this department who helped to assess the sections.

References

1 Sternberger LA. Immunocytochemistry. Englewood Cliffs: Prentice Hall, 1974.

2 Heyderman E. Immunoperoxidase technique in histopathology: applications, methods and controls. $J$ Clin Pathol 1973;32:971-8.

3 Bourne JA. Handbook of immunoperoxidase staining methods. Santa Barbara: Dako Corporation, 1983.

Requests for reprints to: Dr I M Muir, Department of Surgery, St Vincent's Hospital, Fitzroy, Victoria 3065, Australia.

buffered glutaraldehyde. A standard prepolymerisation schedule, taking four and a half hours, was used. Medium hardness EMIX resin and EMcap embedding capsules (EMscope Ltd) were used. Control polymerisation was achieved by heating overnight (16 hours) in a $70^{\circ} \mathrm{C}$ oven, while tests were carried out in a Sharp microwave oven (Model R4060) of $400 \mathrm{~W}$ output rating at $2450 \mathrm{MHz}$. All timings were undertaken manually as the intrinsic timer was too inaccurate for the short time intervals concerned.

The two primary tissue samples chosen for this study had been fixed for more than 24 hours. An additional fresh tissue sample was subjected to $60 \mathrm{sec}-$ onds of microwave assisted fixation in glutaraldehyde, according to the technique of Leong $e t$ $a l^{2}$ before being subjected to the same preparation and polymerisation schedules as the primary test samples. Conventional sectioning staining and photographic procedures were used throughout.

\section{Results}

The microwave field within the oven was found to be inhomogenous, with a particularly strong central zone. Such non-uniformity, although relatively unimportant where large volumes of liquid are being heated in the centre of the oven, is of major concern when using very small volume embedding capsules that can be placed anywhere within the oven. We have not yet made a detailed analysis of field strength and distribution, but we found that consistent and useful results were obtainable with capsules placed in a row at the front of the oven.

Uninterrupted exposure to the centre of the micro- 